\title{
BMJ Open Role and prevalence of impaired awareness of hypoglycaemia in ambulance service attendances to people who have had a severe hypoglycaemic emergency: a mixed-methods study
}

\author{
Edward A S Duncan, ${ }^{1}$ David Fitzpatrick, ${ }^{2}$ Theresa Ikegwuonu, ${ }^{3}$ Josie Evans, ${ }^{4}$ \\ Margaret Maxwell ${ }^{5}$
}

To cite: Duncan EAS,

Fitzpatrick D, lkegwuonu T, et al. Role and prevalence of impaired awareness of hypoglycaemia in ambulance service attendances to people who have had a severe hypoglycaemic emergency: a mixedmethods study. BMJ Open 2018;8:e019522. doi:10.1136/ bmjopen-2017-019522

- Prepublication history for this paper is available online. To view these files, please visit the journal online (http://dx.doi org/10.1136/bmjopen-2017019522).

Received 7 September 2017 Revised 21 February 2018 Accepted 26 March 2018

\section{Check for updates}

${ }^{1}$ Nursing, Midwifery and Allied Health Professions Research Unit (NMAHP RU), Faculty of Health Sciences and Sport, The University of Stirling, Stirling, UK ${ }^{2}$ Faculty of Health Science and Sport, University of Stirling, Stirling, UK

${ }^{3}$ Institute of Health and Wellbeing, University of Glasgow, Glasgow, UK ${ }^{4}$ Faculty of Health Sciences and Sport, University of Stirling, Stirling, UK

${ }^{5}$ Nursing, Midwifery and Allied Health Professions Research Unit, University of Stirling,

Stirling, UK

Correspondence to Dr Edward A S Duncan; edward.duncan@stir.ac.uk

\section{ABSTRACT}

Objectives (1) To compare the experiences of people who are affected by diabetes-related hypoglycaemia and either do or do not require an emergency attendance and (2) to measure the prevalence of impaired awareness of hypoglycaemia in patients who are attended by an ambulance service due to a severe hypoglycaemic event. Design A sequential mixed-methods study.

Setting A qualitative interview study was undertaken with 31 people with diabetes (types 1 and 2) resident in the central belt of Scotland. A national prevalence survey of 590 Scottish Ambulance Service patients who had recently experienced a severe hypoglycaemic emergency requiring ambulance clinicians attendance. Impaired awareness of hypoglycaemia was measured using two standardised measures.

Results Considerable differences in impaired awareness of hypoglycaemia were found in the experiences of participants who did or did not require the ambulance service to treat their severe hypoglycaemic events. Those who required an ambulance reported fewer warning signs and symptoms. The prevalence of impaired awareness of hypoglycaemia in ambulance service call-outs as assessed by two standardised measures was $53 \%$ and $60 \%$, respectively.

Conclusions The prevalence of impaired awareness of hypoglycaemia among those who require an ambulance following a hypoglycaemic event is more than twice that found in the general population of people with diabetes. This may be because the experiences of impaired awareness in people who require an ambulance following a severe hypoglycaemic event differ to those who do not. This study provides important information to guide future prehospital clinical practice, and to develop and evaluate theoretically informed interventions. Improvements in prehospital care for this patient population could lead to global improvements in health outcomes and decreased service costs.

\section{BACKGROUND}

Diabetes is a serious long-term condition that people experience when the amount
Strengths and limitations of this study

- Increased understanding of the role of impaired awareness of hypoglycaemia in people who require an emergency ambulance response could provide important information to improve outcomes for this patient group globally.

- The mixed-method study design enables both understanding of the patient experience and objective measurement of impaired awareness of hypoglycaemia in people who require ambulance assistance.

- While data collection for both arms of the study is limited to ambulance service users in Scotland, its implications have global relevance.

of glucose in their blood is raised over a prolonged period of time. The prevalence of diabetes is increasing, with $5 \%$ of UK adults now affected. ${ }^{1-3}$ There are two main types of diabetes. Type 1 diabetes (T1D) is an autoimmune disorder that results in the body being unable to produce insulin. Type 2 diabetes (T2D) is caused when a person is unable to produce sufficient insulin, or their insulin does not function properly resulting in a build-up of blood glucose. It is caused by a combination of genetic and environmental factors. Hypoglycaemia, where blood glucose levels fall below $4 \mathrm{mmol} / \mathrm{L},{ }^{4}$ is a common consequence of insulin-treated and sulphonylurea-treated diabetes. Mild hypoglycaemia leads to unpleasant symptoms that can impact on everyday activities, but it can be self-treated. Severe hypoglycaemia is a serious condition where the individual needs third-party assistance to be treated. ${ }^{4}$ The prevalence of patients with T1D reporting severe hypoglycaemia $(20 \%-25 \%)^{5}$ is more common in insulin-treated T2D $(6 \%),{ }^{6}$ but the incidence of severe hypoglycaemia rises 
to a similar degree with length of duration of insulin or sulphonylurea therapy. ${ }^{5}$ Untreated, severe hypoglycaemia can result in severe morbidity and death. Recurrent episodes of severe hypoglycaemia can cause permanent cognitive impairment, and an acceleration of the onset of dementia. ${ }^{57}$ Severe hypoglycaemic events are not inevitable. Patients often recognise the neuroglycopenic early warning signs (eg, confusion, odd behaviour) and autonomic symptoms (eg, palpitations, perspiration) and take preventative action, However, for some patients, these symptoms become diminished or absent and they are said to have developed a condition known as impaired awareness of hypoglycaemia (IAH). Approximately, 25\% patients with T1D experience IAH. ${ }^{8}$ Impaired awareness of hypoglycaemia is a treatable condition. ${ }^{9}$

There is variation in how severe hypoglycaemic events are managed. While the majority of severe hypoglycaemic events are treated by people's family and friends, some result in an emergency ambulance attendance. Diabetes-related severe hypoglycaemia events account for between 48000 and 98376 UK emergency ambulance calls per year. ${ }^{10}$ The majority of these $(63 \%-73 \%)$ are fully treated and left at the scene. ${ }^{11-13}$ Between $2 \%$ and $7 \%$ of patients require repeat ambulance attendance, within 2 days, due to a recurrent severe hypoglycaemic event. ${ }^{11-17}$ A recent cross-sectional study of short-term outcomes for patients who had experienced a severe hypoglycaemic event and had been attended by ambulance clinicians in South Africa demonstrates that this is a global problem. ${ }^{18}$

Understanding why some people who have a severe hypoglycaemic event require ambulance service assistance is vital to improve this patient group's health outcomes and service delivery. People who experience a severe hypoglycaemic event should attend their general practitioner (GP) or diabetes specialist for diabetes management advice and a review of their medication with the aim of reducing the probability of a recurrent event. Exploratory qualitative research with 26 participants who had been attended by ambulance clinicians due to a hypoglycaemic event indicated that impaired awareness of hypoglycaemia may be a contributing factor that was not recognised by participants as part of their condition. ${ }^{19}$ As a result participants may have felt there was little need to follow up their care due to their perceived inevitability of such events. The prevalence and experience of impaired awareness of hypoglycaemia among patients who require ambulance attendance has not previously been reported.

Based on the above considerations, this study sought to: (1) compare the experiences of people who are affected by diabetes-related hypoglycaemia and either do or do not require an emergency attendance and (2) measure the prevalence of impaired awareness of hypoglycaemia in patients who are attended by an ambulance service due to a severe hypoglycaemic event. This knowledge could assist in the development of an effective intervention to improve patient follow-up, reduce hypoglycaemic emergencies attended by ambulance services, and lead to improved patient outcomes and cost savings.

\section{METHODS}

\section{Overall design}

We employed a sequential mixed-method design. We first conducted longitudinal qualitative semistructured interviews with two groups of people with diabetes: those who had been attended by an ambulance due to hypoglycaemia, and those who experienced hypoglycaemia but had not required ambulance attendance. These findings indicated that impaired awareness of hypoglycaemia may be an explanatory factor for why some people require assistance from the ambulance service, while many others do not. As a result of the qualitative interview findings, we conducted a national cross-sectional survey to measure the prevalence of impaired awareness of hypoglycaemia in patients who had been attended by an ambulance due to a severe hypoglycaemic event. This paper has been prepared in line with Strengthening the Reporting of Observational Studies in Epidemiology ${ }^{20}$ and COnsolidated criteria for REporting Qualitative research ${ }^{21}$ reporting guidelines.

\section{Patient and public involvement statement}

The longitudinal semistructured interview study questions were developed in consultation with three volunteers from Diabetes UK (Scotland). Two females and one male volunteer representing a wide age range (24-67 years) commented on and revised the content of the interview topic guide. Diabetes UK (Scotland) assisted in the recruitment of participants to the study. The prevalence survey study was informed by patient responses within the longitudinal study, but there was no patient involvement in the survey's design. A lay summary of this paper will be made available to Diabetes UK (Scotland) and disseminated using social media.

\section{Longitudinal qualitative semistructured interview study \\ Study setting}

The setting of the qualitative interview study was the urban central belt of Scotland, UK. Participants were recruited through (1) The Scottish Ambulance Service (SAS) and (2) Diabetes Scotland and the University of Stirling to identify an ambulance service using population, and a population that had not used the ambulance service due to a hypoglycaemic emergency. Participants were purposively sampled to maximise diversity according to their sociodemographic characteristics as measured by the Scottish Index of Multiple Deprivation (SIMD). The SIMD scores are based on seven domains (income, employment, education, housing, health, crime and geographical access), and can be identified using people's postal codes (The Scottish Government 2009). This study used SIMD quintile scores which range from 1 to 5 ( 1 being most deprived and 5 least deprived).

\section{Data collection}

A purposive sampling strategy was used. This enabled comparison of recent emergency service users (SAS participants) and those who had not recently used 
Table 1 Qualitative interview inclusion/exclusion criteria and recruitment strategy

\begin{tabular}{|c|c|}
\hline Inclusion/exclusion criteria & Recruitment strategy \\
\hline $\begin{array}{l}\text { Inclusion criteria: } \\
\text { > Selfyears of age. } \\
\text { - Resident in the central belt of Scotland. } \\
\text { Treated with insulin therapy. } \\
\text { Experience a self-identified hypoglycaemic episode at least } \\
\text { once a month. } \\
\text { Exclusion criteria: } \\
\text { Inability to communicate in English. } \\
\text { Participants lacking capacity to consent. }\end{array}$ & $\begin{array}{l}\text { SAS sample. } \\
\text { Patients who had experienced a hypoglycaemic emergency } \\
\text { in the preceding week were identified via the SAS electronic } \\
\text { patient record system. They were initially contacted by phone } \\
\text { and sent information about the study if they expressed } \\
\text { an interest in knowing more about the study. Potential } \\
\text { participants were asked to confirm interest in the study. } \\
\text { A suitable date and time for the first interview was then } \\
\text { arranged. Participants were given a further opportunity to ask } \\
\text { further questions immediately prior to the interview before } \\
\text { consenting to participate. } \\
\text { Non-SAS sample. } \\
\text { Participants were predominantly recruited through Diabetes } \\
\text { Scotland. Diabetes Scotland distributed study flyers and } \\
\text { posted a study advert on their social media pages. Interested } \\
\text { people contacted the research team and were sent study } \\
\text { information. Participants were also recruited through an } \\
\text { advertisement placed on the web portal of the University of } \\
\text { Stirling. }\end{array}$ \\
\hline
\end{tabular}

SAS, Scottish Ambulance Service; T1D, type 1 diabetes; T2D, type 2 diabetes.

emergency services (non-SAS participants, primarily recruited through Diabetes UK Scotland). Inclusion/ exclusion criteria and recruitment methods are detailed in table 1.

Participants took part (August 2011 to July 2012) in one semistructured interview, lasting about 60 minutes and three shorter bimonthly telephone interviews over 6 months. The semistructured interviews took a pragmatic perspective and aimed to understand participants' experiences of hypoglycaemia and gain an understanding of different individual explanations for their hypoglycaemic episodes. Interviews were recorded using a digital voice recorder. Interviewees were accompanied by a family member or friend when requested. Data collection procedures were piloted with three volunteers (age: 24-67; female $n=2$, male $n=1$ ), and minor adjustments were made to the interview topic guide.

Interviews were conducted by a female social scientist (TI) as part of her doctoral studies. Bespoke training in in-depth interviewing was provided to TI by EASD and MM prior to the commencement of data collection. Participants were aware that the interviews were being carried out as part of the interviewer's doctoral studies. They had no relationship with the researcher prior to the study.

\section{Analysis}

Interviews were audio-recorded, transcribed verbatim, entered into NVivo V.9 and analysed using framework analysis. ${ }^{2}$ Interview recordings were listened to and transcripts read several times in order to become closely familiar with the data and to develop emerging themes. ${ }^{2}{ }^{3} \mathrm{~A}$ thematic framework was developed (by TI) from five transcripts selected to ensure a variety of participants' experiences. This initial framework was cross-checked by two other researchers (EASD and $\mathrm{MM}$ ) and further refined through critical discussion. The development of the final framework was an iterative process undertaken by three researchers (TI, EASD, MM) with regular discussions to ensure consistency in indexing data (as conducted by TI). Charting involved abstraction and synthesis of the indexed data. Data were then summarised, abstracted and synthesised according to their thematic reference and charted to the appropriate part of the thematic framework. Finally, the charts, abstracts and summaries were used to develop and refine concepts and to map associations between themes. ${ }^{22}$ It is not possible to state with certainty that the identified themes reached theoretical saturation, however, the derived themes appear comprehensive, parsimonious and complete.

\section{Prevalence survey study}

Study setting

A national survey of impaired awareness of hypoglycaemia was conducted across Scotland, UK. Patients over 16 years of age who required assistance from the SAS for a diabetes-related emergency with a blood glucose less than $4 \mathrm{mmol} / \mathrm{L}$ between January 2016 and June 2016 were identified as potential participants by a monthly review of ambulance service calls. Patients were not included in the survey if they were resident in secure units, or did not have a residential address.

\section{Data collection}

Potential participants were posted a study pack containing a covering letter, a copy of the questionnaire, a study pen and a prepaid response envelope. 
Each questionnaire had a unique study number. A master sheet linking questionnaires to participants was held within the ambulance service. Reminder study packs were sent to all non-respondents after a fortnight. Consent to participate was assumed through completion and return of the questionnaire.

The questionnaire had two parts. Part 1 contained demographic questions regarding the patient and asked about their diabetes treatment. Part 2 comprised a composite survey comprising the two standardised and validated questionnaire measures of participants' impaired awareness of hypoglycaemia: The Gold ${ }^{23}$ method and the Clarke ${ }^{24}$ Hypoglycaemic Awareness Survey. The Clarke ${ }^{24}$ measure comprised multiple questions that were aggregated into a single score of impaired awareness. It therefore takes longer to complete. The Gold method consists of a single 7 point (1-always aware; 7- never aware) Likert scale question, 'Do you know when your hypos are commencing'? ${ }^{23}$ Both measures were included as we were uncertain which would be more suitable for patients to self-complete in a prehospital setting.

\section{Analysis}

An a priori target sample size of 499 was set to allow us to estimate proportions of impaired awareness of hypoglycaemia within a 5\% margin of error and a 99\% CI. A previous survey ${ }^{10}$ of patients who had used the ambulance service due to a severe hypoglycaemic event gained a $35 \%$ response rate after delivery of a study questionnaire and follow-up reminder. Therefore, we expected to require questionnaires to be sent to 1200 potential participants to obtain sufficient responses for our analysis to have statistical power.

Both the Gold method ${ }^{23}$ and the Clark Hypoglycaemic Awareness Survey ${ }^{24}$ are valid and reliable scales for measuring impaired awareness of hypoglycaemia. A score of 4 or more on either measure indicates the presence of impaired awareness of hypoglycaemia. Data were entered onto SPSS V.21 for analysis. Missing data were assumed to be missing at random. Differences in the mean IAH scores between demographic and treatment groups were assessed using the two-sample t-test or the $\chi^{2} /$ Fisher's exact test.

\section{RESULTS}

Longitudinal qualitative semistructured interview

Participant characteristics

Thirty participants took part in the qualitative interviews (table 2). No participants dropped out of the interview study. Participant's SIMD ratings were spread across all five deprivation categories. However, there was a clustering of participants on both ends of the scale: $57 \%$ of respondents were in deprivation category 4 or 5 (very little deprivation), while $36 \%$ were in the deprivation categories 1 or 2 (higher levels of deprivation). Interestingly, 14 of the 17 non-SAS participants were in deprivation categories 4 or 5 , which is reflected in a mean score of 4. SAS participants tended to live in more deprived areas, with a mean deprivation score of 2.4.

Major differences were evident in participants' experiences of hypoglycaemia between non-ambulance service using and ambulance service using participants. Several of the ambulance service using participants described how they could feel 'perfectly normal' even though their blood glucose level was very low and stated that a lack of awareness of hypoglycaemia symptoms had resulted in them requiring repeat ambulance attendances:

It [hypos] just happens just like that and if you're not onto it right away you're in trouble. (AS3_M14)

At some points I was taking two and three [severe hypoglycaemic events] a day, that's how bad things had got. [...] the biggest majority of them have been where the paramedics have been out and had to bring me back, blood sugars below zero... below 1.1 and all that, this is how bad it had got. You get the paramedic out in the morning and get them back out at night time again. (AS2_M25)

Some participants mentioned that their hypoglycaemic episodes came on so quickly it left them little time to identify the hypoglycaemic episode and treat themselves:

Lately it has been, what, about three times hasn't it I've been into the... with the ambulance, you know. (AS3_M14)

In comparison to ambulance service participants, the other participants spoke about the theme of needing external help much less. None of the non-ambulance service participants needed ambulance service care during the study.

Table 2 Qualitative participant characteristics

\begin{tabular}{|c|c|c|c|c|c|c|c|}
\hline \multirow[b]{2}{*}{ Group } & \multirow[b]{2}{*}{$\mathbf{n}$} & \multirow[b]{2}{*}{ Mean age (SD) } & \multicolumn{2}{|c|}{ Gender } & \multicolumn{2}{|c|}{ Diabetes type } & \multirow{2}{*}{$\begin{array}{l}\text { Diabetes duration } \\
\text { Years (SD) }\end{array}$} \\
\hline & & & Males & Females & T1D & T2D & \\
\hline $\begin{array}{l}\text { Qualitative: ambulance } \\
\text { service }\end{array}$ & 13 & $44(13.87)$ & 6 & 7 & 9 & 4 & 28 (15.39) \\
\hline $\begin{array}{l}\text { Qualitative: non- } \\
\text { ambulance service }\end{array}$ & 17 & $50(17.20)$ & 8 & 9 & 15 & 2 & $21(16.68)$ \\
\hline
\end{tabular}

T1D, type 1 diabetes; T2D, type 2 diabetes. 


\section{Awareness}

A commonly reported phenomenon perception was the loss of early warning symptoms of an impending severe hypoglycaemic emergency. These changes could occur over time, or occasionally due to changes in medication:

When I used to be able to feel it was like two-ish [refers to blood glucose] and that's when I was, like, getting really tingly lips and stuff like that, that's when I knew it was really down low. The lowest I've... when I've actually been pregnant, the lowest I've been is, like, 1.5 I think it was, it was really low, but I didn't have any warning with that one, it was quite low and not to get warning. (AS1_F22)

The new insulins, the human insulins make the reactions a little bit less and much quicker, so you haven't got a lot of time to muck about, you know finding is this high, is this low, is this whatever. So I generally, if I'm feeling odd at all, I'll test my blood, sometimes I'm testing about 10 times a day, but it's just to be sure, you've got the blood glucose monitoring equipment, it's silly to guess when you don't need to. (NS2_F01)

\section{Impact of hypoglycaemic episodes}

The extent to which hypoglycaemic episodes impacted on participants' everyday life depended on the severity of the event, the response and how long recovery took. There was considerable variation regarding the severity of hypoglycaemic episodes participants experienced. Many non-ambulance service using participants described hypoglycaemic episodes as a 'minor inconvenience' that they usually treated themselves.

It is an interruption, but it's not a major thing really for me with my lifestyle. I know how to get over it, know how to deal with it, I do that quickly as I can and move on. So it's not a huge thing for me really, a nuisance though, I get annoyed [laugh]! (NS3_F10)

When hypoglycaemic episodes were more severe, the impact on participants' everyday life was far reaching. For example, one 82-year-old ambulance service using participant spent 2 weeks in hospital following a severe hypoglycaemic event:

I got the ambulance right away and they took me straight into hospital, you know, and I was in there for a fortnight. (AS3_M14)
The impact of recurrent hypoglycaemic episodes

Several participants identified changes in their hypoglycaemia symptoms over time. These often appeared associated with impaired awareness of hypoglycaemia, which in turn was linked to frequency and severity of hypoglycaemic events. Changes in frequency of hypoglycaemic events were mentioned by both groups. Non-ambulance service using participants spoke about this issue less and generally stated that they were experiencing fewer hypoglycaemic episodes than previously. Participants related their decreased frequency of hypoglycaemic events with better diabetes control, which was a result of better health education and learning from experience.

I probably think I had more hypos five years ago because I was taking higher amounts of insulin, whereas I don't think I'm having as many and I know how to deal with them now more than what I did five years ago. Whereas before I was just in a bit of denial about it all, and it's just helped being more regulated, sort of, in the past couple of years about things, so I know what's going to happen and if I get one I can explain it, whereas back then I probably couldn't explain what was happening. (NS1_F08)

In contrast, all ambulance service using participants who mentioned changes in frequency concluded that they were experiencing more hypoglycaemic episodes than previously. Common reasons that participants attributed this increase to include a reduced ability to control diabetes, being unaware of hypoglycaemic events commencing and duration of diabetes:

I seem to be having more hypos. It's probably because I'm not detecting the signs soon enough (AS2_M28) I might have had more since I retired, erm... maybe that's because of the length of time I've had diabetes, I don't... I think you get less warning the longer you have it. Less warning of a hypo. (AS3_F24)

\section{National prevalence survey}

One thousand seven hundred and eighty-seven hypoglycaemia calls were attended by the ambulance service during the 6-month study period. These calls accounted for 1526 patients. Questionnaires were returned from 590 people $(590 / 1526,39 \%)$. Respondents were older than non-respondents $(\mathrm{t}(1790)=4.612, \mathrm{p}<0.005))$. There were no statistically significant differences between responders and non-responders on gender or on SIMD. Characteristics of respondents are shown in table 3 .

Table 3 Survey participant characteristics

\begin{tabular}{|c|c|c|c|c|c|c|c|c|}
\hline \multirow[b]{2}{*}{$\mathbf{n}$} & \multicolumn{2}{|l|}{ Mean age (SD) } & \multicolumn{3}{|l|}{ Gender } & \multicolumn{3}{|c|}{ Diabetes type } \\
\hline & Years mean (SD) & Missing & Males (\%) & Females (\%) & Missing & T1D & T2D & Missing \\
\hline 590 & 61.74 (17.737) & 5 & $325(55)$ & $255(43)$ & 10 & $376(63.70)$ & 200 (34.70) & $14(2.40)$ \\
\hline
\end{tabular}

T1D, type 1 diabetes; T2D, type 2 diabetes. 
Table 4 Participants' impaired awareness of hypoglycaemia

\begin{tabular}{llllllll} 
& \multicolumn{1}{l}{ Gold } & & & \multicolumn{2}{l}{ Clarke } \\
& Normal & IAH & Missing & & Normal & IAH & Missing \\
\hline No $(\%)$ & $267(45.25)$ & $315(53.39)$ & $8(1.35)$ & & $197(39.95)$ & $297(60.12)$ & 97 \\
\hline
\end{tabular}

Participants' responses on the treatments they took to control their diabetes showed that most respondents controlled their diabetes by taking insulin in isolation (T1D $\mathrm{n}=335 / 376$; T2D $\mathrm{n}=74 / 200$ ), or in combination with dietary measures (T1D $n=4 / 376$; T2D $n=4 / 200$ ). Some reported taking tablet medication in isolation (T1D $\mathrm{n}=3 / 376$; T2D $\mathrm{n}=49 / 200$ ), in combination with insulin (T1D 20/376; T2D n=43/200) or with diet control (T1D $\mathrm{n}=0 / 376$; T2D $\mathrm{n}=10 / 200)$. A few participants reported controlling their diabetes using all three approaches (T1D $n=5 / 376$; T2D $n=12 / 200$ ). Very few participants reported controlling their diabetes through diet alone (T1D $n=1 / 376$; T2D $n=6 / 200$ ).

The prevalence of impaired awareness of hypoglycaemia using Gold $^{23}$ and Clarke ${ }^{24}$ methods was 53.39\% and $60.12 \%$, respectively (table 4 ). A moderate association between the Gold ${ }^{23}$ and Clarke ${ }^{24}$ scores of impaired awareness of hypoglycaemia was found $\left(r_{s}=0.509, p \leq 0.01\right)$.

\section{DISCUSSION}

Our qualitative study findings indicated considerable differences between non-ambulance service using participants, who mostly reported only experiencing mild hypoglycaemic events, and participants who required ambulance clinicians to treat severe hypoglycaemic events. In contrast to participants who were not attended by an ambulance clinician, participants who received an ambulance clinician intervention often described a lack of warning signs and symptoms preceding their hypoglycaemic emergency. Participants who required the ambulance service reported an increase in frequency of hypoglycaemic emergencies over the study period, while participants who did not require an ambulance reported experiencing improved glycaemic control and fewer hypoglycaemic events. The prevalence survey results of impaired awareness of hypoglycaemia are consistent with our qualitative findings and indicate that people who require ambulance support for a hypoglycaemic emergency are more than twice as likely to have impaired awareness of hypoglycaemia. These findings have important theoretical, clinical and research implications.

There are good theoretical reasons that may explain participants' perceived lack of importance in attending a GP or diabetes specialist nurse following a hypoglycaemic emergency. Leventhal's common sense model of illness self-regulation ${ }^{25}$ may explain why many participants appeared resigned to the inevitability of severe hypoglycaemic events and therefore did not follow up their care or develop any coping strategy. Participants' appeared to perceive their hypoglycaemia to be a condition of short duration, treated quickly and with few significant side effects. Association with any underlying condition, such as impaired awareness of hypoglycaemia, was therefore lacking. Leventhal's model suggests that such an absence of perceived illness identity may contribute to participants' lack of follow-up care behaviours. ${ }^{25}$ The apparent influence of impaired awareness of hypoglycaemia as a contributory factor to severe hypoglycaemic events in people who require an ambulance may provide a means by which patients' illness identity could be increased. ${ }^{26}$ Impaired awareness of hypoglycaemia is treatable. Identifying and informing patients who have this condition could lead to improvements in patient's illness perception, increased likelihood of patients following up their care after a severe hypoglycaemic event and consequently lead to improvements in glycaemic awareness and control. This, in turn, could lead to better health outcomes and fewer ambulance service calls.

Identifying impaired awareness of hypoglycaemia in patients in the prehospital setting is highly feasible if a brief measure is used. The Clarke ${ }^{24}$ measure takes longer to complete, and in our study many of these questions were not answered by participants. However, completion of the Gold method, that consists of a single Likert scale question, was high. Ambulance clinicians are required to undertake a number of clinical assessments before determining a patient's suitability for non-conveyance. Beyond standard physiological measures such as blood glucose $>5 \mathrm{mmol} / \mathrm{L}$, other measures such as Glasgow Coma Score (GCS) and patient's capacity are formally assessed and recorded. For individuals without capacity or with a reduced GCS an impaired awareness of hypoglycaemia measure should not be applied. However, for those who are deemed to have capacity and a GCS of 15 ambulance clinicians could use the Gold measure after normal glycaemic levels have been re-established, without significantly lengthening their time on scene. If the results indicate that the patient may be experiencing impaired awareness of hypoglycaemia then they could be informed of this, and the importance of attending their GP or diabetes nurse specialist further reinforced to address this treatable condition. The costs of delivering this additional component of care would be negligible, while the potential health benefits and cost savings could be substantial.

The prevalence of impaired awareness of hypoglycaemia in patients who have had a severe hypoglycaemic event that has been attended by ambulance clinicians could meaningfully inform future intervention development and evaluation. A previous intervention study 
to improve levels of patient self-referral demonstrated good patient and clinician acceptability, but an initial linked data analysis suggested it had no impact on patient follow-up behaviour. ${ }^{10}$ Adding ambulance clinician assessment of impaired awareness of hypoglycaemia and immediate reinforcement of the treatability of this condition is now theoretically and empirically supported and could provide an important component of a revised intervention.

\section{Limitations}

Non-ambulance service participants in the qualitative study were largely drawn from the membership of Diabetes UK Scotland. This population is arguably more informed regarding their condition than a more general diabetic population. This may have had an effect on both participants' responses in their interview and on their diabetes-related health behaviours. Due to time limitations, transcripts and overall findings were not returned to participants for correction or comment. Survey responders were slightly older than non-responders. As incidence of impaired awareness of hypoglycaemia increases with age, this may have had an impact on the reported prevalence of impaired awareness of hypoglycaemia. However, the age differences were slight so this is unlikely to have had a large effect on our results. We did not collect information in our questionnaire on diabetes duration or disease-specific information such as prescribed glucose lowering medications. We recognise that such information would have been beneficial and added further detail to our findings. However, we wished to avoid lengthening the questionnaire and potentially decreasing our response rate. The qualitative and quantitative data were collected 5 years apart. This is unlikely to have had any effect on the outcomes of the study as there is no indication or theoretical reason that the timing of the studies influenced the findings, and there have been no significant changes in prehospital emergency treatment of hypoglycaemia in the intervening period.

\section{CONCLUSIONS}

Our study was the first to demonstrate that the prevalence of impaired awareness of hypoglycaemia in patients who are attended by an ambulance service clinician due to a severe hypoglycaemic event is more than double that which is found in the general diabetic population. This finding provides an understanding of why some people require an ambulance to assist with a severe hypoglycaemic event, while the majority do not. This knowledge can be used to shape future prehospital clinical practice and empirical and theory-based intervention development globally.

\section{Recommendations}

Practice

The Gold assessment should be incorporated into ambulance clinician's assessment of patients who have had a severe hypoglycaemic emergency where appropriate. Patients who score $\geq 4$ should be advised: (1) they may be experiencing impaired awareness of hypoglycaemia; (2) that impaired awareness of hypoglycaemia is treatable, and if dealt with is likely to decrease their frequency of severe hypoglycaemic emergencies and (3) that they should therefore make an appointment to follow up their ambulance care with their usual diabetes care provider.

\section{Research}

An optimised intervention to improve referral for diabetes care following a hypoglycaemic emergency that incorporates the assessment and referral of patients who are found to have impaired awareness of hypoglycaemia should be developed and tested.

Acknowledgements The authors would like to thank Jane-Claire-Judson, Allan Kirkwood, Honor Shaw, Stephen Fyfe and the volunteers of Diabetes UK (Scotland) for their help in the design of and recruitment to the longitudinal interview study; all participants for their time and contribution to this study and Catherine Best for providing statistical support.

Contributors The studies were coconceived by EASD, TI, DF and MM. All the authors contributed to elements of the study design. JE contributed expert advice to the design and quantitative analysis of the survey. TI and DF completed data collection. TI led the qualitative analysis. EASD led the quantitative analysis. All of the authors participated in the interpretation of the data. EASD led on the drafting of the manuscript. All authors revised the manuscript for important intellectual content, and read and approved the final manuscript.

Funding The study was funded by the Scottish Ambulance Service and the University of Stirling.

Competing interests None declared.

Patient consent Not required.

Ethics approval Ethical approval for both the qualitative and survey studies were received from the National Research Ethics Service (NRES) (11/AL/0330 and 15/ $\mathrm{EE} / 0383)$. ENHS research and development approval was gained from the Scottish Ambulance Service.

Provenance and peer review Not commissioned; externally peer reviewed.

Data sharing statement Both datasets are available from the corresponding author. There is no unpublished additional data.

Open Access This is an Open Access article distributed in accordance with the Creative Commons Attribution Non Commercial (CC BY-NC 4.0) license, which permits others to distribute, remix, adapt, build upon this work non-commercially, and license their derivative works on different terms, provided the original work is properly cited and the use is non-commercial. See: http://creativecommons.org/ licenses/by-nc/4.0/

(C) Article author(s) (or their employer(s) unless otherwise stated in the text of the article) 2018. All rights reserved. No commercial use is permitted unless otherwise expressly granted.

\section{REFERENCES}

1. Holman N, Young B, Gadsby R. Current prevalence of Type 1 and Type 2 diabetes in adults and children in the UK. Diabet Med 2015;32:1119-20.

2. Ritchie J, Spencer L. Qualitative data analysis for applied policy research. The qualitative researcher's companion 2002;573:305-29.

3. Ritchie J, Spencer L, O'Connor W. Carrying out qualitative analysis. Qualitative research practice: a guide for social science students and researchers 2003;98:219-62.

4. Seaquist ER, Anderson J, Childs B, et al. Hypoglycemia and diabetes: a report of a workgroup of the American Diabetes Association and the Endocrine Society. J Clin Endocrinol Metab 2013;98:1845-59.

5. Frier BM. Hypoglycaemia in diabetes mellitus: epidemiology and clinical implications. Nat Rev Endocrinol 2014;10:711-22. 
6. Edridge CL, Dunkley AJ, Bodicoat DH, et al. Prevalence and incidence of hypoglycaemia in 532,542 people with type 2 diabetes on oral therapies and insulin: a systematic review and meta-analysis of population based studies. PLoS One 2015;10:e0126427.

7. Graveling AJ, Frier BM. Impaired awareness of hypoglycaemia: a review. Diabetes Metab 2010;36 Suppl 3:S64-S74.

8. Geddes J, Schopman JE, Zammitt NN, et al. Prevalence of impaired awareness of hypoglycaemia in adults with Type 1 diabetes. Diabet Med 2008;25:501-4.

9. Hepburn DA, Patrick AW, Eadington DW, et al. Unawareness of hypoglycaemia in insulin-treated diabetic patients: prevalence and relationship to autonomic neuropathy. Diabet Med 1990;7:711-7.

10. Duncan EA, Fitzpatrick D. Improving self-referral for diabetes care following hypoglycaemic emergencies: a feasibility study with linked patient data analysis. BMC Emerg Med 2016;16:13.

11. Brackenridge A, Wallbank H, Lawrenson RA, et al. Emergency management of diabetes and hypoglycaemia. Emerg Med $J$ 2006;23:183-5

12. Farmer AJ, Brockbank KJ, Keech ML, et al. Incidence and costs of severe hypoglycaemia requiring attendance by the emergency medical services in South Central England. Diabet Med 2012;29:1447-50.

13. Khunti K, Fisher H, Paul S, et al. Severe hypoglycaemia requiring emergency medical assistance by ambulance services in the East Midlands: a retrospective study. Prim Care Diabetes 2013;7:159-65.

14. Cain E, Ackroyd-Stolarz S, Alexiadis P, et al. Prehospital hypoglycemia: the safety of not transporting treated patients. Prehosp Emerg Care 2003; 7:458-65.

15. Holstein A, Egberts EH. Risk of hypoglycaemia with oral antidiabetic agents in patients with Type 2 diabetes. Exp Clin Endocrinol Diabetes 2003:111:405-14.

16. Mattila EM, Kuisma MJ, Sund KP, et al. Out-of-hospital hypoglycaemia is safely and cost-effectively treated by paramedics. Eur J Emerg Med 2004;11:70-4.
17. Parsaik AK, Carter RE, Pattan V, et al. Population-based study of severe hypoglycemia requiring emergency medical service assistance reveals unique findings. J Diabetes Sci Technol 2012;6:65-73.

18. Ridhaa Booley M, Welzel T. A cross-sectional analysis of the short-term outcomes of patients receiving prehospital treatment for symptomatic hypoglycaemia in Cape Town. Afr J Emerg Med 2015;5:159-64

19. Fitzpatrick D. Hypoglycaemic emergencies attended by the Scottish Ambulance Service: a multiple methods investigation: University of Stirling, 2015.

20. Vandenbroucke JP, von Elm E, Altman DG, et al. Strengthening the Reporting of Observational Studies in Epidemiology (STROBE): explanation and elaboration. PLoS Med 2007;4:e297.

21. Tong A, Sainsbury P, Craig J. Consolidated criteria for reporting qualitative research (COREQ): a 32-item checklist for interviews and focus groups. Int J Qual Health Care 2007;19:349-57.

22. Mays N, Pope C. Qualitative research in health care: Wiley Online Library, 1996.

23. Gold AE, MacLeod KM, Frier BM. Frequency of severe hypoglycemia in patients with type I diabetes with impaired awareness of hypoglycemia. Diabetes Care 1994;17:697-703.

24. Clarke WL, Cox DJ, Gonder-Frederick LA, et al. Reduced awareness of hypoglycemia in adults with IDDM. A prospective study of hypoglycemic frequency and associated symptoms. Diabetes Care 1995;18:517-22.

25. Leventhal H, Leventhal EA, Contrada RJ. Self-regulation, health, and behavior: a perceptual-cognitive approach. Psychol Health 1998;13:717-33.

26. Rogers HA, de Zoysa N, Amiel SA. Patient experience of hypoglycaemia unawareness in Type 1 diabetes: are patients appropriately concerned? Diabet Med 2012;29:321-7. 